4CPS-270 A SIMULATION PILOT STUDY OF HEALTH OPTIMISATION FOR PATIENTS WITH BIPOLAR DISORDERS: AN EMERGING ROLE FOR CLINICAL PHARMACISTS AS DECISION COACHES

${ }^{1} \mathrm{LM}$ Seljeflot*, ${ }^{2} \mathrm{HD}$ Nguyen, ${ }^{3} \varnothing$ Eiring. ${ }^{1}$ Hospital Pharmacy Enterprise South-Eastern Norway, Hospital Pharmacy Enterprise Gjoevik, Gjoevik, Norway; ${ }^{2}$ University of Glasgow, School of Computing Science, Singapore, Singapore; ${ }^{3}$ Norwegian Institute of Public HealthOslo, Innlandet Hospital Trust, Brumunddal, Norway

\subsection{6/ejhpharm-2019-eahpconf.419}

Background Bipolar disorder patients may experience suboptimal treatment due to ineffective medicines, overtreatment, adverse drug reactions (ADRs) and non-adherence. To ensure optimal and value-congruent treatment for each patient, a holistic approach to shared decision-making (SDM) supported by technologies has been proposed. Our health optimisation system (DECIDE, https:/www.decidetreatment.org) enables patients, healthcare providers and carers to collaborate in selecting, managing, assessing and following up treatment. In SDM, a decision coach is an emerging role in informing, supporting and guiding patients, and clinical pharmacists might be uniquely positioned for the role.

Purpose To explore the role of the pharmacists as decision coaches and to simulate the implementation of a health optimisation system in a pilot study.

Material and methods A literature review and qualitative interviews with psychiatrists and patients were conducted, and multidisciplinary focus groups were applied to establish the pharmacists' role, to produce training programmes and to design a simulation pilot study. We conducted a role-play simulation, to mimic the clinical setting, with eight healthcare professionals. Clinical courses normally taking years were streamlined to 2 weeks using simulation. We then conducted focus groups and semi-structured interviews based on activity theory.

Results The literature review revealed that the role of pharmacists as decision coaches had yet to be fully explored. Based on the qualitative interviews and focus group discussions, pharmacists as coaches could collect patients' medication history, perform a structured medication management review, check medical records and patients' beliefs about the effects and ADRs of medicines used. The pharmacists could enter the obtained information into the DECIDE. The pharmacists could educate, support and follow-up the patient in benefiting from the DECIDE. The participants found that a decision coach could result in a higher quality of treatment and save time. In addition, they generally found the training programme useful, and believed that the role-play simulation could facilitate implementation of the DECIDE in the clinical ward.

Conclusion The role of pharmacists as decision coaches was perceived to be potentially useful and feasible. Further clinical studies are being planned to assess the feasibility of the DECIDE, supported by pharmacists as decision coaches in a clinical setting.
REFERENCES AND/OR ACKNOWLEDGEMENTS

No conflict of interest.

\section{CPS-271 DEVELOPMENT OF PHARMACIST MEDICATION REVIEW IN PAEDIATRIC DISCHARGE PROCESS}

${ }^{1} \mathrm{M}$ Solano*, ${ }^{1} \mathrm{M}$ Jeannin, ${ }^{2} \mathrm{R}$ Anxionnat, ${ }^{1,3} \mathrm{AL}$ Clairet, ${ }^{1,3} \mathrm{~S}$ Limat. ${ }^{1}$ University Hospital of Besançon, Pharmacy Department, Besancon, France; 'University Hospital of Besançon, Paediatric Unit, Besancon, France; ${ }^{3}$ University of Bourgone Franche Comte, Inserm 1098, Besancon, France

\subsection{6/ejhpharm-2019-eahpconf.420}

Background Paediatric patients need follow-up during discharge as they are at higher risk of medication errors owing to complex medical care.

Purpose This study aimed to assess the frequency and type of pharmacist interventions during medication review at paediatric transition from hospital to home. The second aim was to assess patients' comprehension and satisfaction.

Material and methods This was a prospective pilot study conducted by the pharmacy department in a paediatric unit. A pharmacist provided discharge counselling for patients with chronic diseases and introduction or change of treatment during hospitalisation. He conducted follow-up telephone encounters between day 3 and day 7 post-discharge. The number and type of pharmacist interventions and physician acceptance rates were assessed. Patients' comprehension and need for further information were compared before and after pharmacist medication review. The time to obtain treatment after discharge was reported. Patients' satisfaction was identified.

Results There were 41 pharmacist medication reviews during the 7 month study. A pharmacist was able to provide discharge counselling for $49 \%$ of discharges. The pharmacist identified 23 interventions, of which 87\% were accepted and $13 \%$ were informational in nature. The most frequently identified interventions included dosage form optimisation and administration optimisation. An average of patients' knowledge self-assessment was $5.8 / 10$ and $8.6 / 10$ before and after pharmacist discharge counselling, respectively. Patients needed further information concerning administration and side effects for $71 \%$ and $51 \%$ before pharmacist discharge counselling, respectively. After pharmacist discharge counselling, they needed this information for $5 \%$ and $7 \%$, respectively. Seventy-eight per cent of patients could get their treatment without delay after discharge. Eighty-three per cent of patients recommended this type of pharmacist medication review (17\% not provided).

Conclusion Pharmacists can provide a valuable service in patients' management during childrens' discharge process by detecting prescription errors, optimising administration and counselling patients. Facilitating the discharge process satisfies patients and can help to provide continuity of care.

\section{REFERENCES AND/OR ACKNOWLEDGEMENTS}

No conflict of interest. 